\title{
CHALLENGES OF GATED MYOCARDIAL PERFUSION SPECT PROCESSING
}

\author{
Victor Ploskikh \\ Saint-Petersburg State University \\ Russia \\ v.ploskikh@spbu.ru
}

\author{
Elena Kotina \\ Saint-Petersburg State University \\ Russia \\ e.kotina@spbu.ru
}

Article history:

Received 06.09.2021, Accepted 25.11.2021

\begin{abstract}
The paper provides an in-depth look at gated myocardial perfusion single photon emission computed tomography (SPECT) data processing. Attention paid to several unmentioned subjects of the quantitative analysis of gated myocardial perfusion SPECT data. The article considers several options in the construction process of the ellipsoid coordinate system of the left ventricle (LV). Mathematical representation of polar maps is given. Formulas of the regional parameters calculation are proposed. Issues of phase analysis are explored.
\end{abstract}

\section{Key words}

Gated single photon emission computed tomography (gSPECT), mathematical modeling, myocardial perfusion imaging, software, image segmentation

\section{Introduction}

The radionuclide method of studying the internal organs and systems of the human body is a modern method of many disease diagnostics. Digital processing of radionuclide studies is an important stage in obtaining qualitative and quantitative diagnostic information [Kotina and Ploskikh, 2012; Kotina et al., 2013b]. Currently, there is a trend towards automation of research and obtaining accurate quantitative variables [Slomka et al., 2017; Slomka et al., 2016; Gomez et al., 2018; Garcia et al., 2007].

Nuclear cardiology is one of the main fields of nuclear medicine practice. Gated myocardial perfusion SPECT allows investigating the perfusion and function of the heart muscle. Processing of the study includes many stages [Dorbala et al., 2018]: from tomographic reconstruction [Kotina et al., 2013a] and motion correction [Ovsyannikov et al., 2013] to polar map construction and phase analysis [Chen et al., 2005; Hsu et al.,
2013; Babin and Kotina, 2014]. A large number of publications are devoted to the data processing. In the articles [Kotina and Pasechnaya, 2014; Kotina et al., 2019; Bazhanov et al., 2018], methods of image processing based on the construction of the velocity field are developed. A number of papers discuss issues of the myocardium segmentation [Xu et al., 2009; Germano et al., 2016], the parametric image construction [Slomka et al., 2017; Gomez et al., 2018; Kuronuma et al., 2021][Germano and Slomka, 2017], software packages for automated quantification [Germano et al., 2007; Ficaro et al., 2007; Garcia et al., 2007], the prognostic value of gated myocardial perfusion SPECT [Nakajima et al., 2017; Kuronuma et al., 2021; Cabrera-Rodríguez et al., 2013].

Quantitative analysis of gated myocardial perfusion SPECT was considered in [Ploskikh and Kotina, 2018]. This paper continues research in this direction, and some challenges of ECG-gated SPECT data processing are discussed, such as the ellipsoid coordinate system calculation, polar map representation, regional parameters estimation and circular statistics application.

\section{Gated Myocardial Perfusion SPECT}

Radionuclide myocardial perfusion imaging (MPI) with single-photon emission computed tomography (SPECT) is the widely used technique for detecting ischemic heart disease in clinical practice.

Myocardial perfusion tomoscintigraphy synchronized with the ECG signal (gSPECT) provides information on perfusion defects, LV function, regional wall motion, thickening, and dyssynchrony. The accuracy of the quantitative function and perfusion analysis effects on the prognostic impact. Automated quantitation continues to be a challenging field of research. In this article, we consider the principles and give the accurate formulas for the main constructions and quantitations of the most important parameters. 


\subsection{The processing algorithm of myocardial perfu- sion study}

The study is conducted in ECG-gated tomographic acquisition mode [Dorbala et al., 2018].

After the acquisition and reconstruction [Kotina et al., 2013a] a sequence of three-dimensional images $V_{k}, k=$ $1 \ldots N$ is obtained. The images represent information on the distribution of the radionuclide in the LV myocardium at different intervals of the representative cardiac cycle [Kotina et al., 2013b].

Mathematical processing workflow shown on the fig. 1 is thoroughly explained in [Ploskikh and Kotina, 2018].

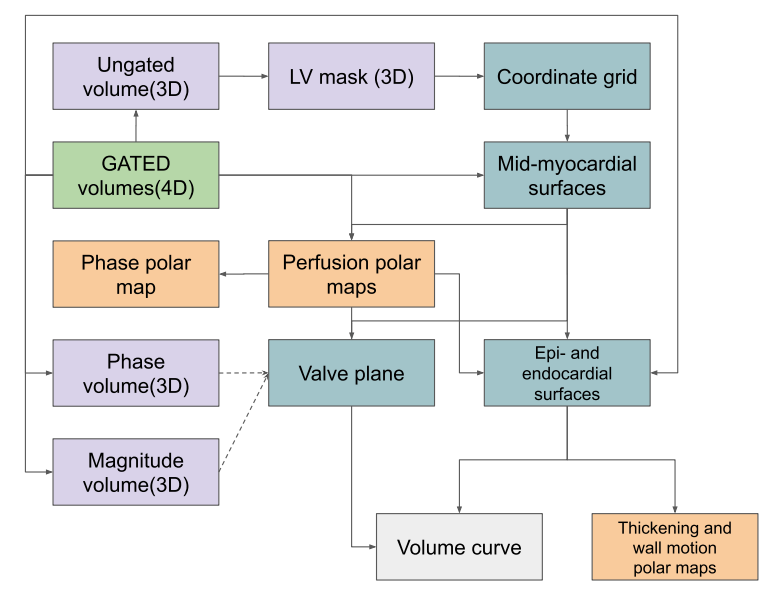

Figure 1. Cardiac gSPECT processing workflow

At the initial stage the phase and magnitude threedimensional images are calculated from the source data. They provide additional information helpful in manual intervention.

The source volumes are averaged to get the ungated image. It is used in segmentation to determine LV voxels. Approximation of voxel positions produces the ellipsoid grid. The ellipsoid is the prolate spheroid with short axis length $R_{s}$ and long axis length $R_{l}$. The grid consists of nodes formed by intersections of latitude and longitude lines. The nodes define the base grid surface that is subsequently used to obtain LV surfaces. All surfaces and parameters are thus bound to the grid nodes.

The mid-myocardial surfaces are determined as offsets from grid node positions along the grid normals. The initial approximation is found with the help of the LoGdetector along the normal profiles of the source perfusion volumes. The surfaces themselves are obtained by optimizing the deviations from the initial approximation and adjacent nodes.

Sampling the gated volumes at nodes of corresponding mid-myocardial surfaces produces perfusion polar maps that are subsequently used to calculate the phase map.

The valve plane is obtained from end-diastolic midmyocardial surface and averaged perfusion map by ap- proximation of underperfused area boundaries.

Epi- and endocardial surfaces construction is carried out by displacing the mid-myocardial surface along its normal. The displacement value is found using a combination of thresholding and optimization methods, local perfusion values, and a condition of constant myocardial volume bound by the valve plane. The functional maps of thickening and wall motion are based on the obtained surfaces.

Volumes constrained by endomyocardial surfaces and valve plane define the volumetric curve which in turn used to calculate hemodynamic parameters.

\subsection{Latitude selection}

The grid is defined as a set of nodes regularly spaced in latitude and longitude. A number of different definitions of latitude are used in geodesy, but little attention is paid to their application in the context of the LV coordinate system. The parametric latitude $\beta$ was used in previous work[Ploskikh and Kotina, 2018]. This paper considers the application of the rectifying latitude.

The rectifying latitude $\mu$ can be defined through the meridian distance to apical pole $l(\beta)$ :

$$
\begin{gathered}
\mu=-\frac{\pi}{2}+\pi \frac{l(\beta)}{l\left(\frac{\pi}{2}\right)}, \\
l(\beta)=\int_{-\frac{\pi}{2}}^{\beta} \sqrt{1+\frac{R_{s}^{2}-R_{l}^{2}}{R_{l}^{2}} \sin ^{2} \theta} d \theta .
\end{gathered}
$$

Fig. 2 shows the distribution of uniformly selected parametric and rectifying latitudes on a prolate spheroid. It can be noted that the parametric latitude lines have a higher density in the vicinity of the poles.

The inverse formula for $\beta(\mu)$ can be defined, but exact implementation is out of scope of this work.

In addition to choosing the type of latitude, two ways to form a uniform distribution of $N_{\text {lat }}$ non-polar latitudes can be defined:

$$
\begin{gathered}
\theta_{1, j}=-\frac{\pi}{2}+\frac{\pi j}{N_{l a t}+1}, j=1 \ldots N_{l a t}, \\
\theta_{2, j}=-\frac{\pi}{2}+\frac{\pi}{N_{l a t}}\left(j-\frac{1}{2}\right), j=1 \ldots N_{\text {lat }} .
\end{gathered}
$$

\subsection{Grid generalization}

Let's introduce the following generalizing parameters into the process of creation of the ellipsoid grid: latitude type (parametric or rectifying), type of latitude distribution $t \in\{1,2\}$, number of non-polar latitudes $N_{\text {lat }}$, number of longitudes $N_{\text {lon }}$, sectoral longitudinal shift $\Delta \in[0,1)$. For consistency with the previous work, 


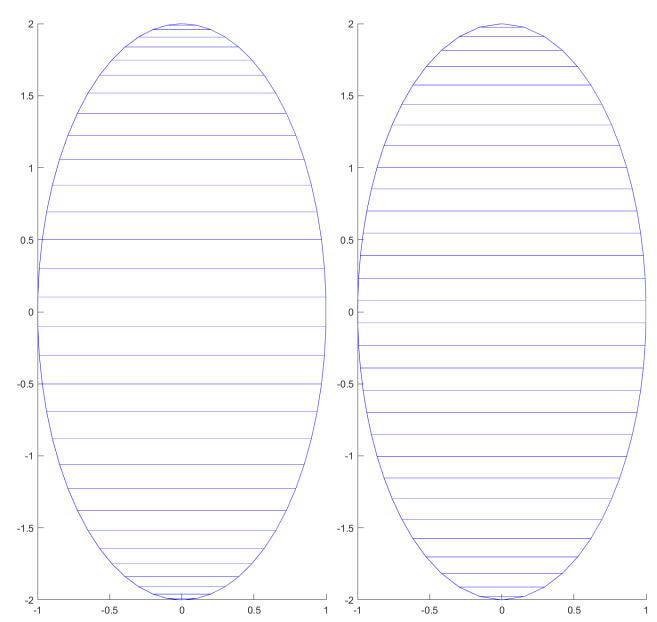

Figure 2. Uniform distribution of parametric (left) and rectifying (right) latitudes

we define the parametric latitude $\beta_{i}$ and longitude $\lambda_{i}$ of $\left(N_{\text {lat }} N_{\text {lon }}+2\right)$ grid nodes as follows:

$$
\begin{gathered}
\beta_{i}=\left\{\begin{array}{cc}
\beta\left(\theta_{t,\left\lfloor(i-1) / N_{l o n}\right\rfloor+1}\right) & \text { if rectifying } \\
\theta_{t,\left\lfloor(i-1) / N_{l o n}\right\rfloor+1}, & \text { if parametric }
\end{array},\right. \\
\mu_{i}=\left\{\begin{array}{cc}
\theta_{t,\left\lfloor(i-1) / N_{l o n}\right\rfloor+1} & \text { if rectifying } \\
\mu\left(\theta_{t,\left\lfloor(i-1) / N_{l o n}\right\rfloor+1}\right), & \text { if parametric }
\end{array},\right. \\
\lambda_{i}=\frac{2 \pi}{N_{l o n}}(i-1+\Delta),
\end{gathered}
$$

for non-polar nodes where $i=1 \ldots N_{\text {lat }} N_{\text {lon }}$, and

$$
\begin{gathered}
\beta_{N_{\text {lat }} N_{\text {lon }}+1}=\mu_{N_{\text {lat }} N_{\text {lon }}+1}=-\frac{\pi}{2} \\
\beta_{N_{\text {lat }} N_{\text {lon }}+2}=\mu_{N_{\text {lat }} N_{\text {lon }}+2}=\frac{\pi}{2} \\
\lambda_{N_{\text {lat }} N_{\text {lon }}+1}=\lambda_{N_{\text {lat }} N_{\text {lon }}+2}=0
\end{gathered}
$$

for poles.

The next section discusses the effect of these parameters on the computation of regional values on polar maps.

\section{Polar maps}

The ellipsoid grid allows us to extract a standard number of data samples $S_{i}, i=1 \ldots\left(N_{l a t} N_{l o n}+2\right)$ regardless of myocardial size. These samples represent the state of distinct regions of the myocardium and can be displayed two-dimensionally as a polar map.

\subsection{Polar map definition}

A polar map is based on polar azimuthal equidistant projection. The samples are color coded and then placed in unit circle as follows

$$
\left(\begin{array}{c}
x_{i} \\
y_{i}
\end{array}\right)=\frac{l\left(\beta_{i}\right) l\left(\frac{\pi}{2}\right)}{\frac{\pi}{2}+\mu_{\text {bas }}}\left(\begin{array}{c}
\cos \lambda_{i} \\
\sin \lambda_{i}
\end{array}\right),
$$

where $x_{i}^{2}+y_{i}^{2} \leq 1$, and $\mu_{\text {bas }}$ is the basal latitude. Samples with rectifying latitude more than $\mu_{\text {bas }}$ are ignored as they are usually located in the basal area across the valve plane and do not represent myocardium. The selection of the basal latitude is implementation dependent. About two thirds of arc length adjacent to apex are used in practice.

The formula (9) is simplified using the rectifying latitude:

$$
\left(\begin{array}{c}
x_{i} \\
y_{i}
\end{array}\right)=\frac{\frac{\pi}{2}+\mu_{i}}{\frac{\pi}{2}+\mu_{b a s}}\left(\begin{array}{c}
\cos \lambda_{i} \\
\sin \lambda_{i}
\end{array}\right)
$$

Figure 3 shows polar maps based on different types of latitude. An increased density of samples in the apex region can be noted for the parametric case.

\subsection{Regional parameters}

The polar map is divided into 17 or 20 segments (fig. 4) to assess the regional parameters of the myocardium.

The segment is defined via latitudinal $\left(\mu^{-}, \mu^{+}\right)$and longitudinal $\left(\lambda^{-}, \lambda^{+}\right)$bounds. The segment value calculated as weighted average of grid samples:

$$
S_{\text {seg }}=\frac{\sum_{i=1}^{N_{\text {lat }} N_{\text {lon }}} w_{i}^{\text {lat }} w_{i}^{\text {lon }} w_{i}^{\text {are }} S_{i}}{\sum_{i=1}^{N_{\text {lat }} N_{\text {lon }}} w_{i}^{\text {lat }} w_{i}^{\text {lon }} w_{i}^{\text {are }}},
$$

where $w_{i}^{\text {lat }}$ is a latitudinal weight, $w_{i}^{\text {lon }}$ is a latitudinal weight, $w_{i}^{\text {are }}$ is area weight defined by formulas

$$
\begin{aligned}
& w_{i}^{\text {lat }}= \\
& \left\{\begin{array}{c}
0, \quad \mu_{i} \leq \mu^{-}-\Delta \mu_{i} \\
\left.\left(\frac{1}{2}+\frac{\mu_{i}-\mu^{-}}{\Delta \mu_{i}}\right)\right|_{[0,1]}, \mu_{i} \in\left[\mu^{-}-\Delta \mu_{i}, \mu^{-}\right) \\
\left.\left(\frac{1}{2}+\frac{\mu_{i}-\mu^{-}}{\Delta \mu_{i-1}}\right)\right|_{[0,1]}, \mu_{i} \in\left[\mu^{-}, \mu^{-}+\Delta \mu_{i-1}\right) \\
1, \quad \mu_{i} \in\left[\mu^{-}+\Delta \mu_{i-1}, \mu^{+}-\Delta \mu_{i}\right) \\
\left.\left(\frac{1}{2}-\frac{\mu_{i}-\mu^{+}}{\Delta \mu_{i}}\right)\right|_{[0,1]}, \mu_{i} \in\left[\mu^{+}-\Delta \mu_{i}, \mu^{+}\right) \\
\left.\left(\frac{1}{2}-\frac{\mu_{i}-\mu^{+}}{\Delta \mu_{i-1}}\right)\right|_{[0,1]}, \mu_{i} \in\left[\mu^{+}, \mu^{+}+\Delta \mu_{i-1}\right) \\
0, \quad \mu^{+}+\Delta \mu_{i-1} \leq \mu_{i}
\end{array}\right. \\
& \begin{array}{c}
w_{i}^{\text {lon }}=\left.\frac{\lambda_{i}-\lambda^{-}}{2 \Delta \lambda}\right|_{[-1,1]}-\left.\frac{\lambda_{i}-\lambda^{+}}{2 \Delta \lambda}\right|_{[-1,1]} \\
w_{i}^{\text {are }}= \\
\Delta \beta_{i} \sqrt{R_{s}^{2} \cos ^{2} \beta_{i}\left(R_{s}^{2} \sin ^{2} \beta_{i}+R_{l}^{2} \cos ^{2} \beta_{i}\right)}
\end{array}
\end{aligned}
$$




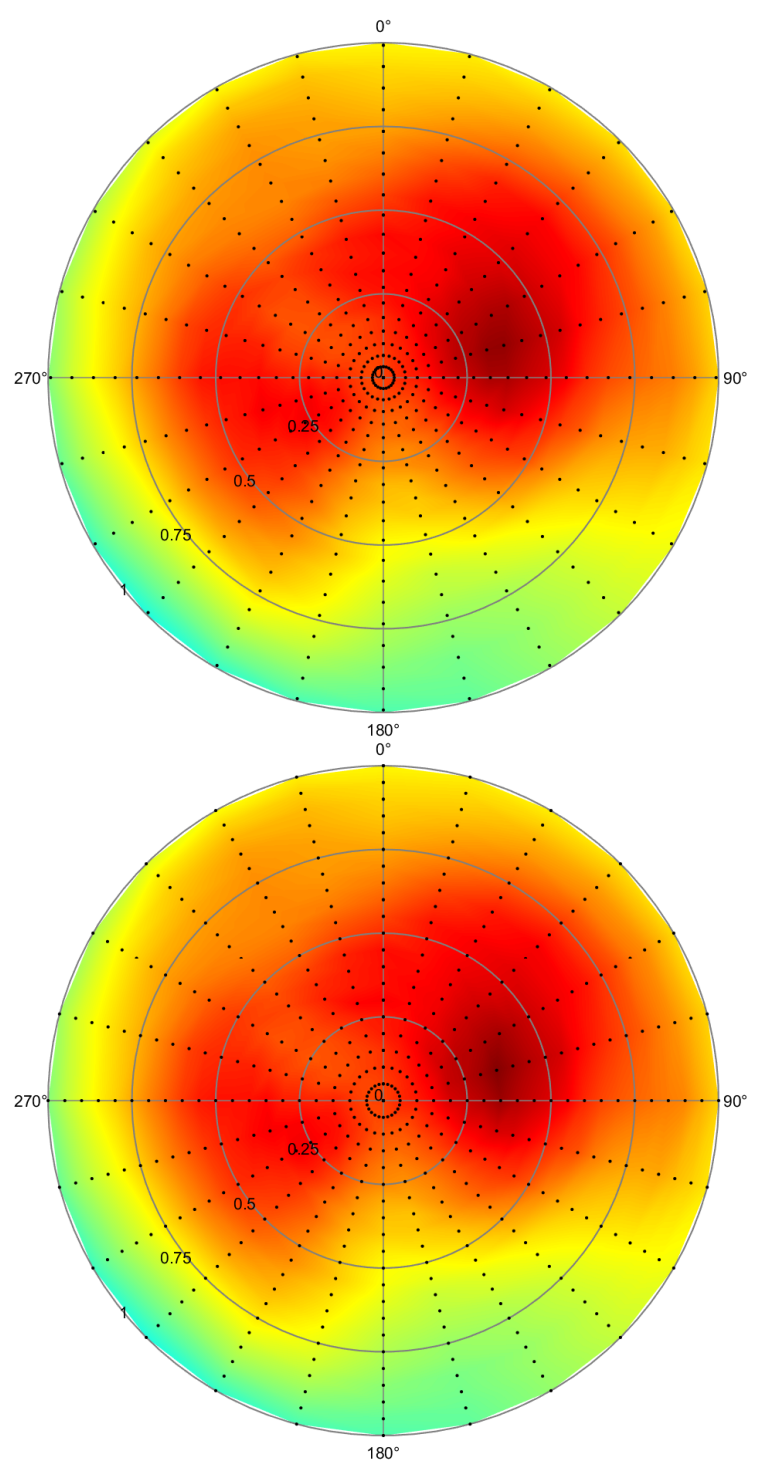

Figure 3. Perfusion polar maps constructed with parametric latitude (top) and rectifying latitude (bottom)

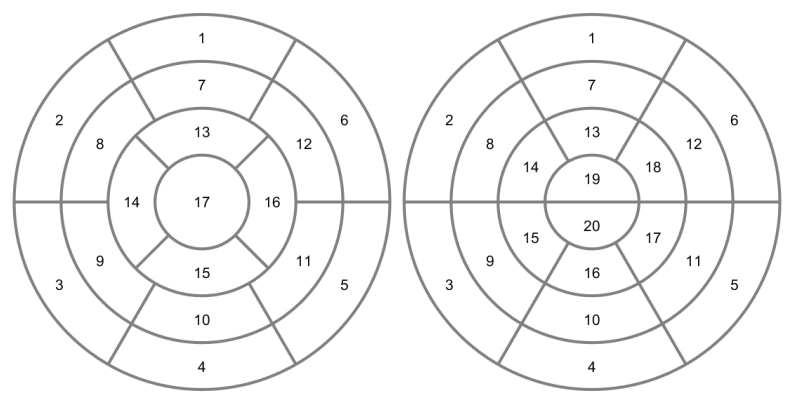

Figure 4. 17-segment (left) and 20-segment(right) models used for semiquantitative visual scoring

where

$$
\left.x\right|_{[a, b]}=\max (a, \min (x, b))
$$

is a clamp operator and

$$
\begin{gathered}
\Delta \mu_{i}=\mu_{\left(i+N_{\text {lon }}\right)}-\mu_{i}, \\
\Delta \lambda=\frac{\pi}{N_{l o n}}, \\
\Delta \beta=\mu_{\left(i+N_{l o n}\right)}-\mu_{\left(i-N_{l o n}\right)} .
\end{gathered}
$$

Figure 3.2 shows that with careful selection of parameters the formulas (12-13) can be written in simpler forms:

$$
\begin{aligned}
& w_{i}^{l a t}=\left\{\begin{array}{l}
1, \mu_{i} \in\left[\mu^{-}, \mu^{+}\right] \\
0, \mu_{i} \notin\left[\mu^{-}, \mu^{+}\right]
\end{array},\right. \\
& w_{i}^{l o n}=\left\{\begin{array}{l}
1, \lambda_{i} \in\left[\lambda^{-}, \lambda^{+}\right] \\
0, \lambda_{i} \notin\left[\lambda^{-}, \lambda^{+}\right]
\end{array},\right.
\end{aligned}
$$

however to compare data from different sources the generalized forms to be used.

\section{Phase}

Phase analysis is a perspective approach as the nonuniform phase distribution is one of the indicators of LV mechanical dyssynchrony. It is based on the phase polar map obtained by the formula:

$$
\phi_{i}=\operatorname{atan} 2\left(b_{i}, a_{i}\right),
$$

where

$$
\begin{aligned}
& a_{i}=\frac{2}{N} \sum_{k=1}^{N} P_{k, i} \cos \frac{2 \pi(k-1)}{N}, \\
& b_{i}=\frac{2}{N} \sum_{k=1}^{N} P_{k, i} \sin \frac{2 \pi(k-1)}{N},
\end{aligned}
$$

and $P_{k, i}$ is the perfusion polar map of $k$-th gating interval.

Since the polar map representation is morphed, it is difficult to objectively determine the size of defects from it. A way to analyze the phase distribution is to represent it in the form of a histogram.

When constructing a histogram, account should be taken of the nonuniform spatial distribution of the grid nodes. To address this issue the phase value at a grid point is taken with an area weight defined by (14).

A small set of analyzed values is usually used in the context of decision-making automation. To analyze the phase uniformity the following distribution parameters are usually selected: mean $(\bar{\phi})$, standard deviation $(\bar{\sigma})$, skewness $(\bar{s})$, excess kurtosis $(\bar{k})$. It should be noted that the phase is a periodic quantity, and therefore standard linear statistics are not suitable for its analysis. Circular statistics should be used, in particular circular mean

$$
\bar{\phi}_{c}=\bar{\phi}_{1}
$$

circular standard deviation

$$
\bar{\sigma}_{c}=\sqrt{-2 \ln \left(\bar{R}_{1}\right)}
$$




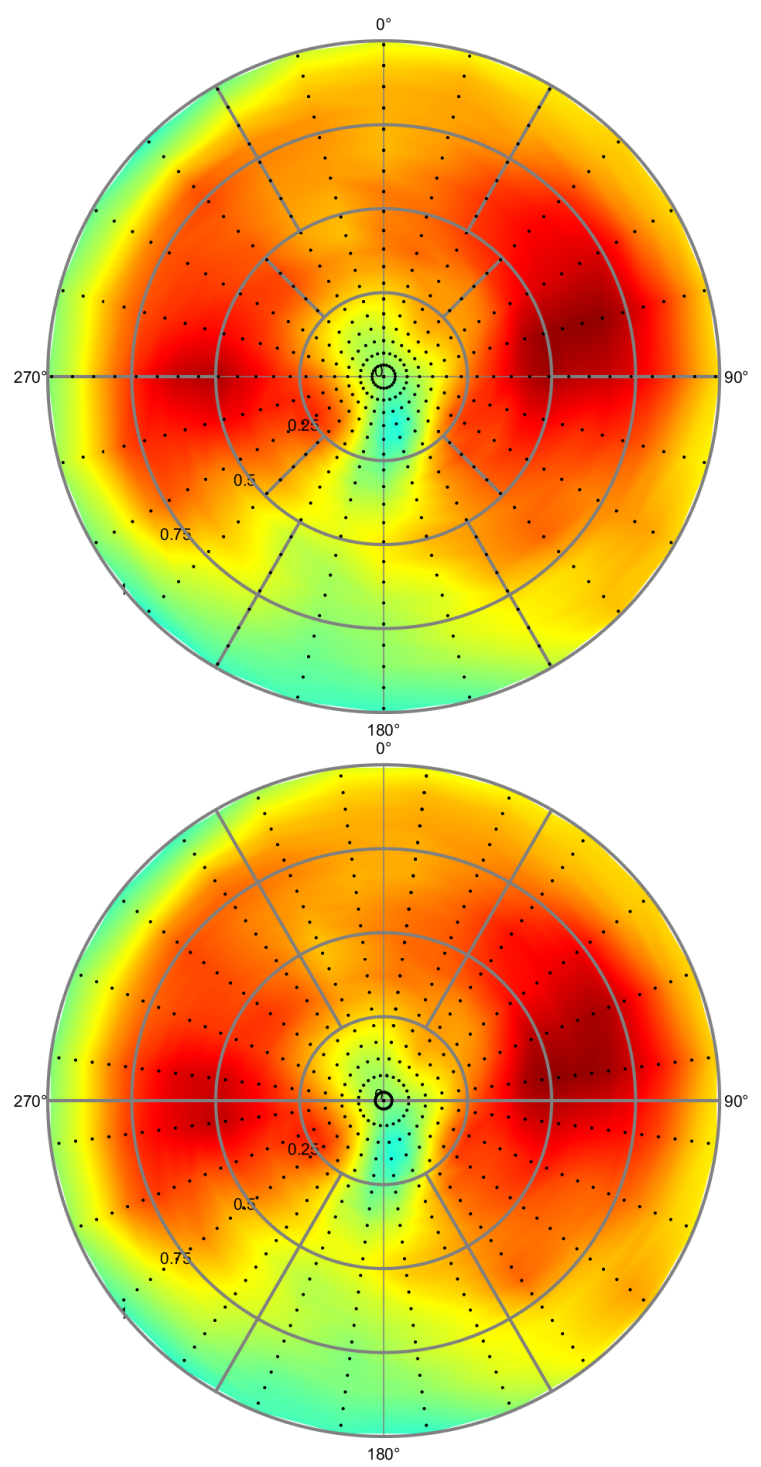

and

$$
\bar{m}_{n}=\frac{\sum_{i=1}^{N_{\text {lat }} N_{\text {lon }}} w_{i}^{\text {lat }} w_{i}^{\text {are }} e^{i \phi_{i} n}}{\sum_{i=1}^{N_{\text {lat }} N_{\text {lon }}} w_{i}^{\text {lat }} w_{i}^{\text {are }}},
$$

is a weighted circular moment of phase distribution on the whole polar map segment $\left(\mu^{-}=0, \mu^{+}=\mu_{\text {bas }}\right)$.

Comparison of different statistics on four real cases is shown in figure 6 and table 1.
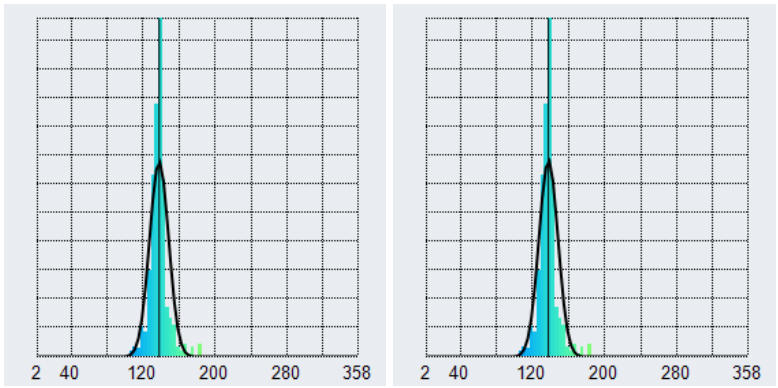

Figure 5. Segmented polar maps $\left(\mu_{\text {bas }}=\frac{\pi}{8}\right)$ :

top - parametric, $N_{\text {lat }}=31, t=1, N_{\text {lon }}=24, \Delta=0$; bot - rectifying, $N_{\text {lat }}=32, t=2, N_{\text {lon }}=24, \Delta=0.5$.
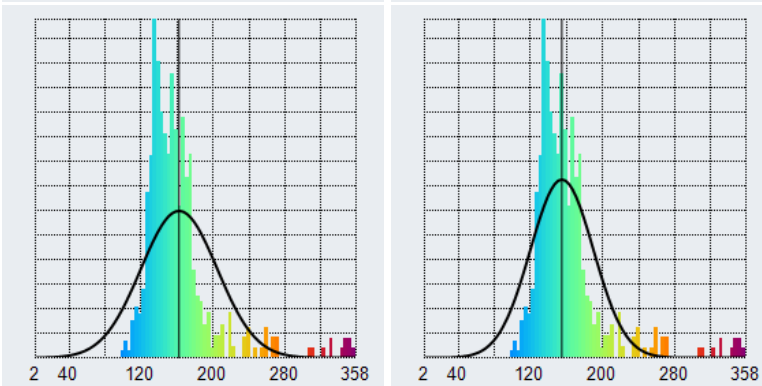

circular skewness

$$
\bar{s}_{c}=\frac{\bar{R}_{2} \sin \left(\bar{\phi}_{2}-2 \bar{\phi}_{1}\right)}{\left(1-\bar{R}_{1}\right)^{3 / 2}}
$$

and circular kurtosis

$$
\bar{k}_{c}=\frac{\bar{R}_{2} \cos \left(\bar{\phi}_{2}-2 \bar{\phi}_{1}\right)-\bar{R}_{1}^{4}}{\left(1-\bar{R}_{1}\right)^{2}}
$$

where

$$
\bar{\phi}_{n}=\operatorname{Arg}\left(\bar{m}_{n}\right)
$$
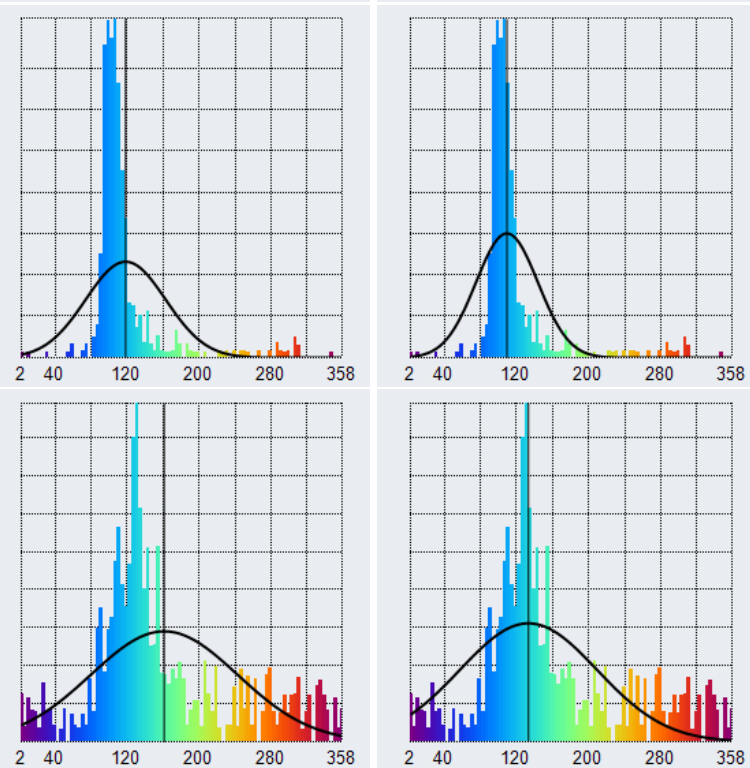

Figure 6. Four cases of phase map histograms are analyzed with conventional (left) and circular (right) statistics

$$
\bar{R}_{n}=\left|\bar{m}_{n}\right|
$$


Table 1. Comparison of statistic values on real cases

\begin{tabular}{c|c|c|c|c}
\hline Statistics & 1 & 2 & 3 & 4 \\
\hline \hline $\bar{\phi}^{\circ}{ }^{\circ}$ & 137 & 161 & 118 & 160 \\
$\bar{\phi}_{c}{ }^{\circ}$ & 137 & 154 & 108 & 132 \\
$\bar{\sigma},{ }^{\circ}$ & 11 & 42 & 44 & 81 \\
$\bar{\sigma}_{c}{ }^{\circ}$ & 11 & 35 & 34 & 76 \\
$\bar{s}$ & 1.1 & 2.5 & 2.7 & 0.66 \\
$\bar{s}_{c}$ & -2.9 & -1.2 & -1.0 & -0.17 \\
$\bar{k}$ & 4.5 & 7.5 & 8.6 & -0.16 \\
$\bar{k}_{c}$ & 8.6 & 7.3 & 11 & 0.99 \\
\hline
\end{tabular}

The following conclusions can be deduced from the results. First, the means and standard deviations have similar values on narrow distributions. Second, on wide distributions linear mean tend to bias toward 180 degrees, and linear standard deviation is greater than circular one. Third, values of skewness and excess kurtosis have bad correlation between linear and circular statistics. In general, the circular means and standard deviations show better behaviour in most cases than its linear counterparts.

\section{Conclusion}

The paper provides an in-depth look into myocardial perfusion gated SPECT data processing. Gated SPECT myocardial perfusion imaging remains one of the most important diagnostic procedures. This article addresses previously unmentioned issues of the algorithm implementation. The algorithm is considered as a data processing workflow. The calculation steps and data flow direction are described. Close attention is paid to several processing stages.

The process of LV ellipsoid coordinate grid construction is generalized. Different latitude definitions are considered. The parameters of the grid construction are introduced. The application of rectifying latitude in combination with the parameters selection helps in simplifying calculation processes.

The definitions of the LV polar map and polar grid segments in terms of the coordinate system are introduced. The formulas of regional parameters calculations are given. Selection of the basal latitude is implementation dependent and remains an open issue.

Special attention is paid to phase polar maps. The application of circular statistics to analyze phase histogram is considered. Test on real data shows that circular statistics have better behaviour in patologic cases. The use of skewness and excess kurtosis don't look promising without further research.

\section{References}

Babin, A. V. and Kotina, E. D. (2014). Mathematical data processing of gated SPECT myocardial perfusion imaging with using wavelet analysis. 2014 International Conference on Computer Technologies in Physical and Engineering Applications, ICCTPEA 2014 Proceedings, pages 19-20.

Bazhanov, P., Kotina, E., Ovsyannikov, D., and Ploskikh, V. (2018). Optimization algorithm of the velocity field determining in image processing . Cybernetics and physics, 7(4):174-181.

Cabrera-Rodríguez, L., Peix, A., Padrón, K., Chacón, D., Carrillo, R., Fernández, Y., and Mena, E. (2013). Prognostic value of gated SPECT after reperfusion for acute myocardial infarction . MEDICC Review, 15(2):20-25.

Chen, J., Garcia, E. V., Folks, R. D., Cooke, C. D., Faber, T. L., Tauxe, E. L., and Iskandrian, A. E. (2005). Onset of left ventricular mechanical contraction as determined by phase analysis of ECG-gated myocardial perfusion SPECT imaging: development of a diagnostic tool for assessment of cardiac mechanical dyssynchrony. J Nucl Cardiol, 12(6):687-695.

Dorbala, S., Ananthasubramaniam, K., Armstrong, I. S., Chareonthaitawee, P., DePuey, E. G., Einstein, A. J., Gropler, R. J., Holly, T. A., Mahmarian, J. J., Park, M. A., Polk, D. M., Russell, R., Slomka, P. J., Thompson, R. C., and Wells, R. G. (2018). Single Photon Emission Computed Tomography (SPECT) Myocardial Perfusion Imaging Guidelines: Instrumentation, Acquisition, Processing, and Interpretation. J Nucl Cardiol, 25(5):1784-1846.

Ficaro, E. P., Lee, B. C., Kritzman, J. N., and Corbett, J. R. (2007). Corridor4DM: the Michigan method for quantitative nuclear cardiology. J Nucl Cardiol, 14(4):455-465.

Garcia, E. V., Faber, T. L., Cooke, C. D., Folks, R. D., Chen, J., and Santana, C. (2007). The increasing role of quantification in clinical nuclear cardiology: the Emory approach. J Nucl Cardiol, 14(4):420-432.

Germano, G., Kavanagh, P. B., Fish, M. B., Lemley, M. H., Xu, Y., Berman, D. S., and Slomka, P. J. (2016). Same-Patient Processing" for multiple cardiac SPECT studies. 1. Improving LV segmentation accuracy. $J$ Nucl Cardiol, 23(6):1435-1441.

Germano, G., Kavanagh, P. B., Slomka, P. J., Van Kriekinge, S. D., Pollard, G., and Berman, D. S. (2007). Quantitation in gated perfusion SPECT imaging: the Cedars-Sinai approach. J Nucl Cardiol, 14(4):433-454.

Gomez, J., Doukky, R., Germano, G., and Slomka, P. (2018). New Trends in Quantitative Nuclear Cardiology Methods. Curr Cardiovasc Imaging Rep, 11(1).

Hsu, T. H., Huang, W. S., Chen, C. C., Hung, G. U., Chen, T. C., Kao, C. H., and Chen, J. (2013). Left ventricular systolic and diastolic dyssynchrony assessed by phase analysis of gated SPECT myocardial perfusion imaging: a comparison with speckle tracking echocardiography. Ann Nucl Med, 27(8):764-771. 
Kotina, E., Leonova, E., and Ploskikh, V. (2019). Radionuclide images processing with the use of discrete systems. Vestnik Sankt-Peterburgskogo Universiteta, Prikladnaya Matematika, Informatika, Protsessy Upravleniya, 15(4):544-554.

Kotina, E. and Pasechnaya, G. A. (2014). Optical flowbased approach for the contour detection in radionuclide images processing. Cybernetics and physics, 3(2):62-65.

Kotina, E. and Ploskikh, V. A. (2012). Data processing and quantitation in nuclear medicine. RuPAC 2012 Contributions to the Proceedings - 23rd Russian Particle Accelerator Conference, pages 526-528.

Kotina, E. D., Latypov, V. N., and Ploskikh, V. A. (2013a). Universal system for tomographic reconstruction on gpus. Problems of Atomic Science and Technology, 88(6):175-178.

Kotina, E. D., Ploskikh, V. A., and Babin, A. B. (2013b). Radionuclide methods application in cardiac studies. Problems of Atomic Science and Technology, 88(6):179-182.

Kuronuma, K., Otaki, Y., Miller, R., Van Kriekinge, S., Sharir, T., Hu, L.-H., Gransar, H., Liang, J., Parekh, T., Kavanagh, P., Einstein, A., Fish, M., Ruddy, T., Kaufmann, P., Sinusas, A., Miller, E., Bateman, T., Dorbala, S., Carli, M., Tamarappoo, B., Dey, D., Berman, D., and Slomka, P. (2021). Prognostic value of automatic phase analysis for predicting adverse cardiac events beyond conventional spect variables: Results from the refine spect. Journal of Nuclear Medicine, 62(supplement 1):27-27.

Nakajima, K., Okuda, K., Matsuo, S., and Slomka, P. (2017). Making the invisible visible: Phase dyssynchrony has potential as a new prognostic marker. $J$ Nucl Cardiol.

Ovsyannikov, D., Kotina, E., and Shirokolobov, A. (2013). Mathematical methods of motion correction in radionuclide studies. Problems of Atomic Science and Technology, 88(6):137-140.

Ploskikh, V. A. and Kotina, E. (2018). Quantitative analysis of gated myocardial perfusion spect . Cybernetics and physics, 7(4):220-227.

Slomka, P., Hung, G. U., Germano, G., and Berman, D. S. (2016). Novel SPECT Technologies and Approaches in Cardiac Imaging. Cardiovasc Innov Appl, 2(1):31-46.

Slomka, P. J., Dey, D., Sitek, A., Motwani, M., Berman, D. S., and Germano, G. (2017). Cardiac imaging: working towards fully-automated machine analysis \& interpretation. Expert Rev Med Devices, 14(3):197212.

Xu, Y., Kavanagh, P., Fish, M., Gerlach, J., Ramesh, A., Lemley, M., Hayes, S., Berman, D. S., Germano, G., and Slomka, P. J. (2009). Automated quality control for segmentation of myocardial perfusion SPECT. $J$. Nucl. Med., 50(9):1418-1426. 